\title{
GMTIFS: The Giant Magellan Telescope integral fields spectrograph and imager
}

\author{
Rob Sharp*a, G. Bloxham ${ }^{\mathrm{a}}$, R. Boz ${ }^{\mathrm{a}}$, D. Bundy ${ }^{\mathrm{a}}$, J. Davies ${ }^{\mathrm{a}}$, B. Espeland ${ }^{\mathrm{a}}$, B. Fordham, ${ }^{\mathrm{a}}$ J. Hart ${ }^{\mathrm{a}}$, N.

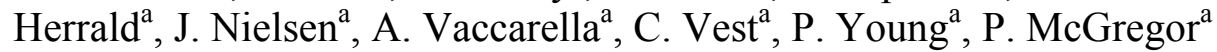 \\ ${ }^{\text {a }}$ Research School of Astronomy \& Astrophysics, The Australian National University, Cotter Road, \\ Weston Creek, ACT 2611, Australia
}

\begin{abstract}
GMTIFS is the first-generation adaptive optics integral-field spectrograph for the GMT, having been selected through a competitive review process in 2011. The GMTIFS concept is for a workhorse single-object integral-field spectrograph, operating at intermediate resolution $(\mathrm{R} \sim 5,000 \& 10,000)$ with a parallel imaging channel. The IFS offers variable spaxel scales to Nyquist sample the diffraction limited GMT PSF from $\lambda \sim 1-2.5 \mu \mathrm{m}$ as well as a 50 mas scale to provide high sensitivity for low surface brightness objects. The GMTIFS will operate with all AO modes of the GMT (Natural guide star - NGSAO, Laser Tomography - LTAO, and, Ground Layer - GLAO) with an emphasis on achieving high skycoverage for LTAO observations. We summarize the principle science drivers for GMTIFS and the major design concepts that allow these goals to be achieved.
\end{abstract}

Keywords: GMT, near-infrared, integral-field spectroscopy

\section{INTRODUCTION}

The Giant Magellan Telescope Integral Field Spectrograph, GMTIFS, was selected as a first generation adaptive optics spectrograph for the Giant Magellan Telescope in 2011 as part of a competitive down select process[1,2]. The instrument concept builds on the earlier successes of the ANU instrumentation program with near-infrared instruments for the Gemini Telescopes, the NIFS ${ }^{[3]}$ AO spectrograph and $\mathrm{GSAOI}^{[4]}$ camera and the highly productive WiFeS ${ }^{[5]}$ IFS for the

ANU $2.3 \mathrm{~m}$ telescope. GMTIFS will provide medium resolution (with $\mathrm{R} \sim 5,000$ and $\mathrm{R} \sim 10,000$ modes) near-infrared $(1<$ $\lambda<2.5 \mu \mathrm{m})$ imaging spectroscopy sampled at the diffraction limit of the AO assisted GMT (10-25 mas) and also at lower angular resolutions (100 mas), over a proportionally wider field of view, for low surface brightness extended sources. An AO assisted Imager arm allows simultaneous observation, at a complementary wavelength, over a field of view well matched to that over which the GMT Adaptive Secondary Mirrors (ASM) delivers corrected imaging.

\section{SCIENCE DRIVERS}

Over the last decade, integral-field spectroscopy has matured from a fringe technique, applicable to only specialist observations, to become a mainstream of observational astronomy. This was due to a number of factors including the maturation of instrument concepts, the availability of robust data processing software and the light-grasp of 8-10 m telescope that provide sufficient signal-to-noise in observations that would otherwise be over resolved at less sensitive facilities. The power of integral-field spectroscopy if further enhanced as adaptive optics on Extremely Large Telescopes (ELTs) begin to access spatial scales appropriate for key physical process not accessible at the $8 \mathrm{~m}$ diffraction limit (or with $H S T$ at shorter wavelengths).

In the ELT era, the most ineresting scientific question are likely to be ones we have not yet formulated, however all robust design processes required a set of concrete design reference programs from which critical requirements can be defined. Those adopted for the GMTIFS are: cosmic time will be studied using kinematic measurements of Ly $\alpha$ and $\mathrm{H} \alpha$ emission that exploit the high sensitivity of the GMTIFS IFS. The broad wavelength coverage of the IFS allows the study of associated chemical evolution of these galaxies via spatially resolved measurements of their rest frame optical emission-line ratios. The high angular resolution of the GMTIFS Imager will be used to probe the

*ob.Sharp@anu.edu.au; +61 (0)2 6125 8035; rsaa.anu.edu.au

Ground-based and Airborne Instrumentation for Astronomy VI, edited by Christopher J. Evans, Luc Simard, Hideki Takami Proc. of SPIE Vol. 9908, 99081Y · (c) 2016 SPIE · CCC code: 0277-786X/16/\$18 · doi: 10.1117/12.2231561 
build-up of the stellar components of these galaxies via broad-band near-infrared imaging. In these ways, GMTIFS will provide essential follow-up observations for the large samples of high-redshift galaxies that will be identified, at lower angular and spectral resolution, with JWST.

- The high angular resolution of the GMTIFS IFS will allow spectroscopic studies of outer exosolar planets identified in near-term Extreme Adaptive Optics surveys on 8-m telescopes. As a first generation AO instrument for GMT, GMTIFS will not offer extreme AO coronagraphy. However, angular and spectral differential imaging will allow sensitive measurements of the newly discovered systems.

- Observations of the near-infrared counterparts of Gamma-Ray Bursts will probe the structure of the intergalactic medium at the epoch of reionization beyond $\mathrm{z} \sim 7$ using the high spectral resolution of the GMTIFS IFS.

- The GMTIFS IFS will probe the most and least massive nuclear black holes in the Universe via high angularresolution measurements of stellar kinematics and Keplerian motions of circumnuclear gas. These observations will clarify the intimate relationship between nuclear black-hole mass and host galaxy stellar properties at the highest and lowest galaxy scale black-hole masses.

- The high angular resolution of the GMTIFS Imager will be used to resolve individual stars in galaxies beyond the Local Group, providing direct insights into the star formation and chemical histories of complex stellar systems in different environments.

\subsection{Galaxy jet dynamics - observing fundamental physical processes}

The high angular resolution and high spectral resolution of the GMTIFS IFS will combine to probe the jet outflows in active galaxies with unprecedented detail. This will reveal the internal structure of the outflows and constrain the launch physics of their jets. Current generation $8 \mathrm{~m}$ telescope AO observations are beginning to show the importance of such interactions in sculpting the formation and evolution of galaxies due to the various feedback processes involved. However, there are limited example systems in the volume currently accessible with sufficient projected spatial resolution $(\leq 1 \mathrm{Kpc})$, and the key epoch at higher redshift $(\mathrm{z}=1-3)$ remains inaccessible at these physical length scales. The light gathering power of the GMT, and the angular resolution it affords with GMTIFS, will open up observations of this critical epoch at physical length scales that allow powerful discrimination between the fundamental models of jet launch and interaction with the host galaxy environment. This process is illustrated via detailed simulations of the performance of GMTIFS using a dedicated data simulation packages (GMTIFSsim, see section 3.2). Based on the magneto-hydrodynamic simulations ${ }^{[6]}$ of a simulated GMT/GMTIFS observation is performed for a young jet source in a host galaxy at a redshift of $\mathrm{z} \approx 2.5$. The simulations results are shown in Figure 1. The extended crown-like structure at the top in the [OIII] emission map is due to the jet driven bubble dredging up matter into the outflow lobe and causing vertical outflows along the jet. The high angular resolution (24 mas at $K$-band) and light gathering power of GMT allows GMTIFS to catch this and other key diagnostic features in action. 


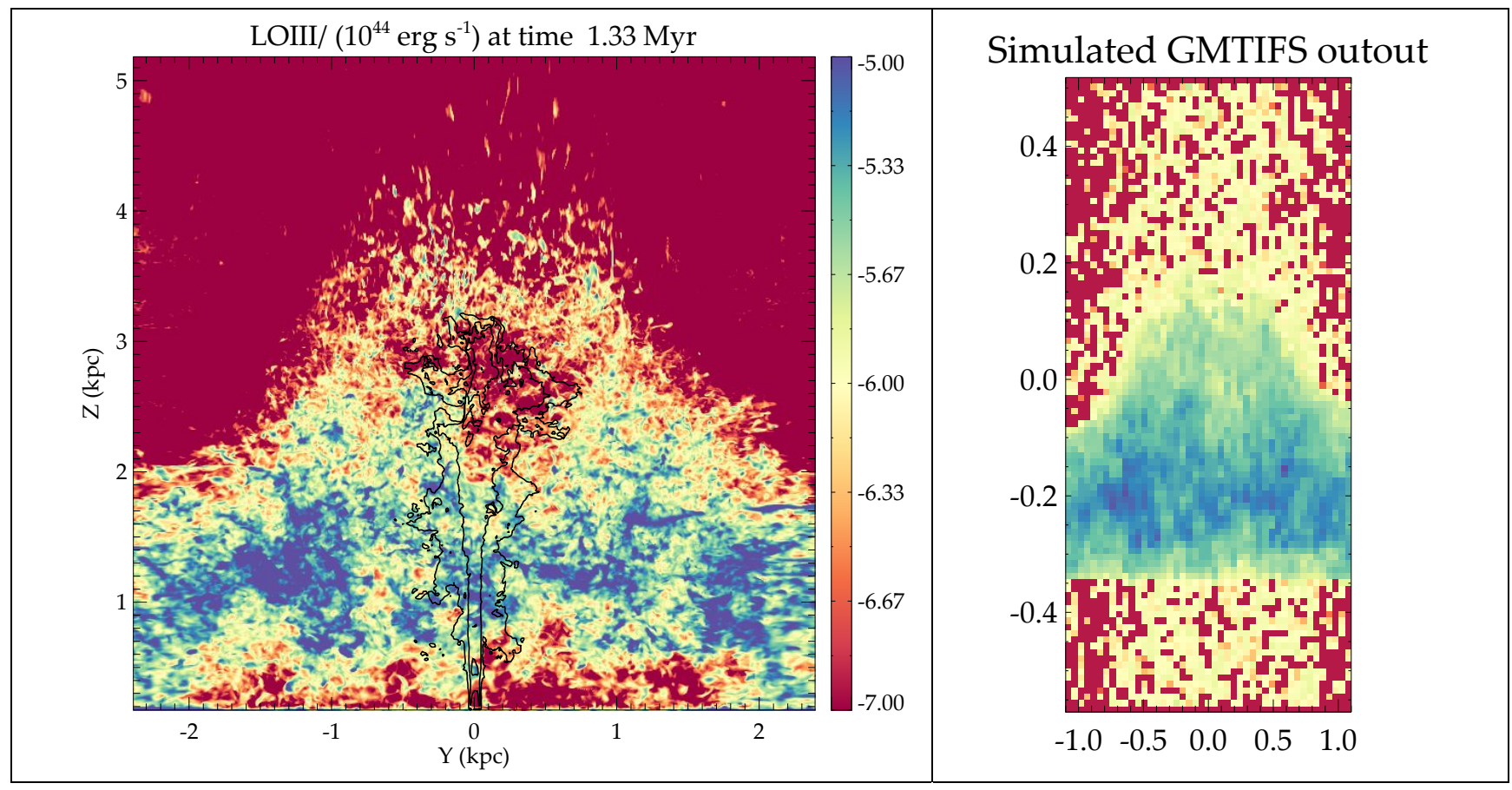

Figure 1: MHD simulations (following Mukherjee ${ }^{[6]}$ et al.) are shown at left at three full input resolution, while the simulated GMTIFS observation ( $\mathrm{R} \sim 10,000,12$ mas spaxels, 3 hours on-source simulated integration) is seen at right. The black contours in the MHD simulated image are the contours of simulated radio intensity at $1.8 \mathrm{GHz}$, indicating the location of the jet.

\section{INSTRUMENT DESCRIPTION}

The GMTIFS project is now mid-way through a Preliminary Design Study. The instrument principles remain little modified from the conceptual design ${ }^{[1]}$ and as such we present only a brief overview in this work and focus instead on design evolution and innovations and refinements. The basic instrument parameters are provided in Table 1 . As a nearinfrared instrument, operating over the wavelength $\lambda=0.95-2.5 \mu \mathrm{m}$, GMTIFS must be housed inside a cooled cryostat to prevent thermal emission dominating the faint astronomical signals. Adopting the requirement that thermal emission from the cryostat body should be subdominant to the intrinsic dark current for the current generation of near-infrared science grade detectors, and adopting a suitable safety margin, a cryostat operating temperature of $\mathrm{T} \leq 120 \mathrm{~K}$, with a stability of $\pm 0.1 \mathrm{~K}$ is sufficient. Detector focal planes will require cooling to $\mathrm{T} \approx 60 \mathrm{~K}$ with a stability requirement of \pm 1 $\mathrm{mK}$. A schematic of the instrument optical train is show in Figure 2 and folded in Figure 3.

\subsection{Fore optics}

The GMT fore optics consist of a number of functional elements. All wavelengths long-ward of $\lambda>0.95 \mu \mathrm{m}$ are transmitted to GMTIFS through the 180 arcsecond diameter $(300 \mathrm{~mm})$ cryostat window. Shorter wavelengths are reflected to the Natural Guide star AO and Laser Tomography AO wavefront sensors hosted on the front of the GMTIFS cryostat. The inclined dichroic window introduces astigmatism that is compensated through the use of a pair of wedged plates $^{[7]}$. A science pick-off fold mirror directs the $20.4 \times 20.4$ arcsec science field to the main instrument while passing the remaining 180 arcsec diameter guide field to the on-instrument wavefront sensor (OIWFS) via a Beam Steering Mirror $(\mathrm{BSM})^{[8,9]}$. The science field is reimaged by a fore optics unit containing a segmented and rotating transmissive colds stop as well as an atmospheric dispersion corrector. A dichroic unit then splits the field in wavelength to feed the IFS with the complementary light passed to the Imager arm. 


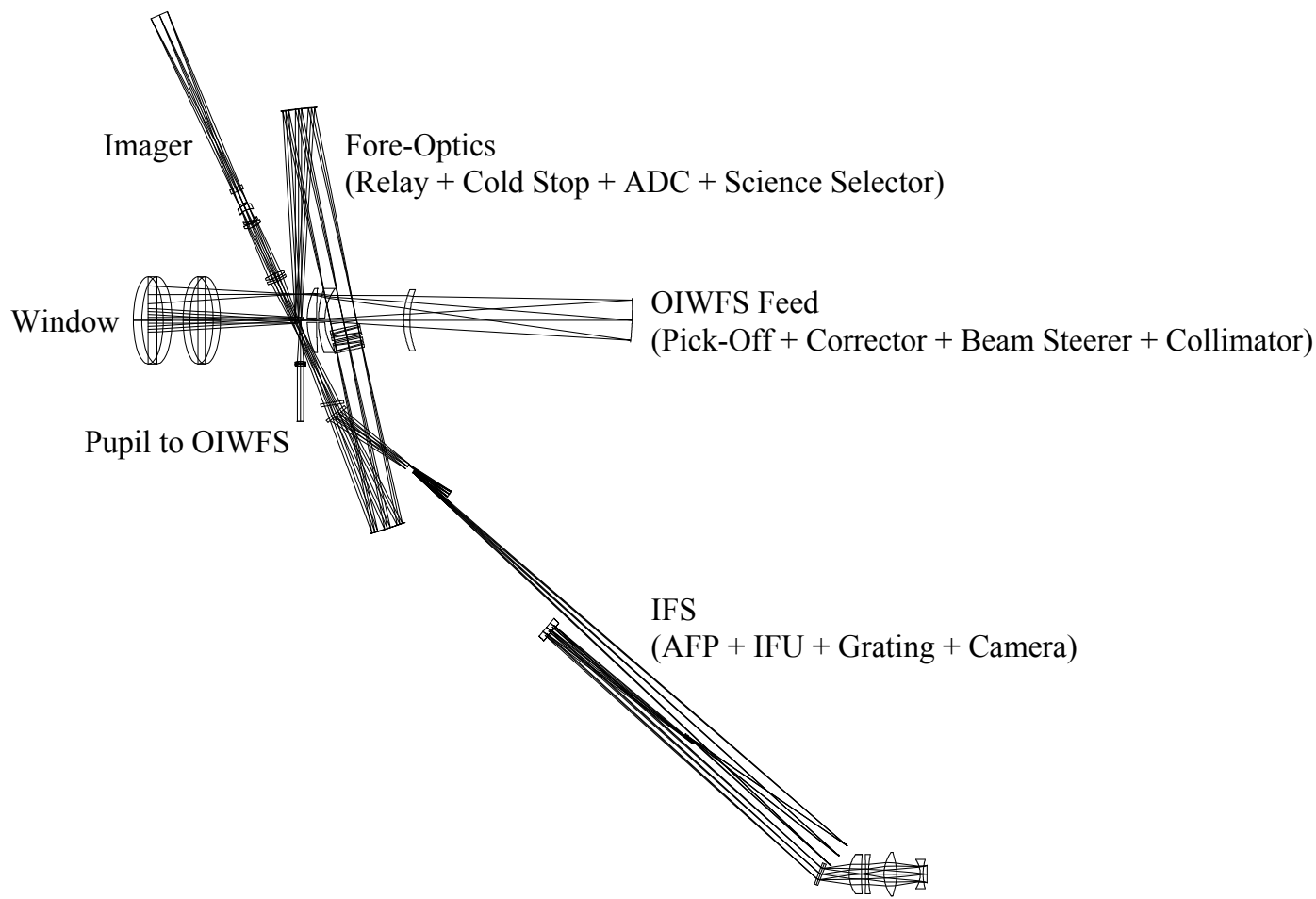

Figure 2: The planar arrangement of the unfolded optical system for GMTIFS.

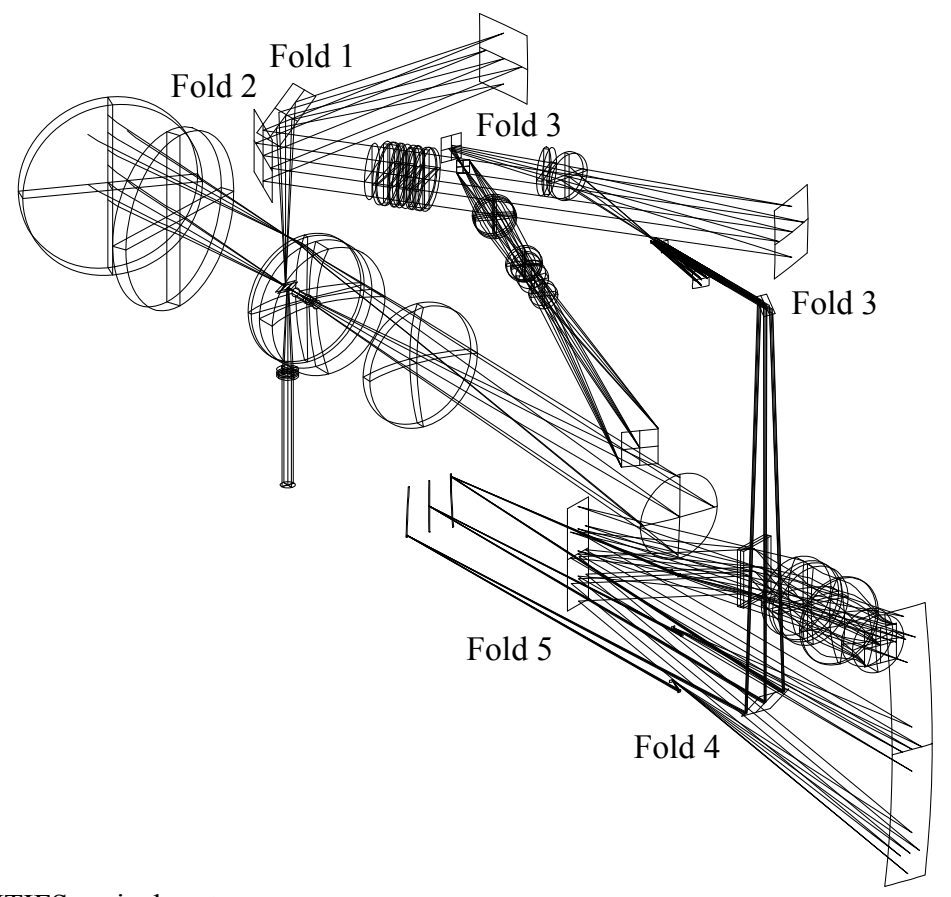

Figure 3: The folder GMTIFS optical system.

\subsection{Integral-Field Spectrograph}

The IFS design builds on the successful Gemini/NIFS ${ }^{[3]}$ and ANU/WiFeS ${ }^{[5]}$ IFS instruments. The concentric image slicer concept $^{[10]}$ employs 45 image slices, each 88 spatial pixels (spaxels) long, and reformatted into a staircase virtual slit. 
The imager slicer unit is fed by one of four deployable anamorphic projection units that deliver one of four interchangeable spatial scales to the common IFU. The anamorphism ensures Nyquist sampling along the slit spatial direction as well as across the slit in the spectral direction, delivering square spaxels on the sky for optimal image packaging onto the available detector real-estate. Since the majority of proposed observations are likely to be close to the redactor readnoise limit when operating between the $\mathrm{OH}$ air-glow emission-lines, not oversampling the spatial PSF provides significant sensitivity gains, while also maximizing the field of view with limited detector real-estate. A rectangular field of view, with a 2:1 aspect ratio permits infield nodding for optimal sky-subtraction efficiency when observing compact sources. A suite of seven interchangeable gratings provides a fixed data format at the detector across each of the atmospheric transmission windows. Two resolution modes are specified, a low-resolution mode, $\mathrm{R} \sim 5,000(\sim$ $\left.60 \mathrm{~km} \mathrm{~s}^{-1}\right)$, sufficient to operate between the $\mathrm{OH}$ airglow lines ${ }^{[11,12]}$ without over-resolving emission lines in extragalactic sources, and high-resolution, $\mathrm{R} \sim 10,000\left(\sim 30 \mathrm{~km} \mathrm{~s}^{-1}\right)$ necessary to probe physical process such as the stellar kinematics in the vicinity if low mass black holes $\left(10^{4}-10^{6} \mathrm{M}_{\odot}\right)$ and nuclear star clusters or outflows in Young stellar objects (YSOs).

Both the IFS and the Imager contain a suite of occulting spots for high-contrast operations although, as noted above, as a first generation AO instrument for GMT, GMTIFS is not intended to operate as an extreme AO coronographic instrument and so its capabilities for high contract imaging are restricted.

Volume Phase Holographic (VPH) gratings are proposed, with attached prisms for beam steering in the high-resolution modes (as for the ANU/WiFeS ${ }^{[5]}$ instrument). Detailed designs for the grating suite have been prepared, however, uncertainty in the availability of the preferred science detector (Teledyne 4096 $\times 4096$ HgCdTe HAWAII-4RG-15 $\mu$ m pixel, see Section 5) has delayed final design specifications and procurement, raising the risk associated with VPH availability.

\section{Data processing}

Despite integral-field spectroscopy becoming more mainstream in recent years, wrangling raw IFS data into science ready products remains a challenge and can hinder early exploitation of IFS instruments. Much of the recent success of the ANU/WiFeS IFS system has been the availability of a high-quality easy-to-use science-ready data pipeline system $\left(\mathrm{PyWiFeS}^{[13]}\right.$ ) capable of delivering science quality processed data in close to real-time (with typically a next day turnaround cycle for most observing and calibration strategies). The small number spectroscopic modes, each with fixed format individually, allows a stable reference data set to be generated to form the basis for rapid and reliable calibration process. Elements of the PyWiFeS package have been integrated into the GMTIFSsim simulation data suite. This simulation tool is being actively used to generate low-level raw GMTIFS data, allowing for accurate exploration of instrument format design decisions, as well as informative predictions about the science made possible with GMT and GMTIFS (see Figure 1). An example of raw and semi-processed data products are shown in Figure 4, while a fully processed simulated observations is compared to its underlying input data in Figure 1.

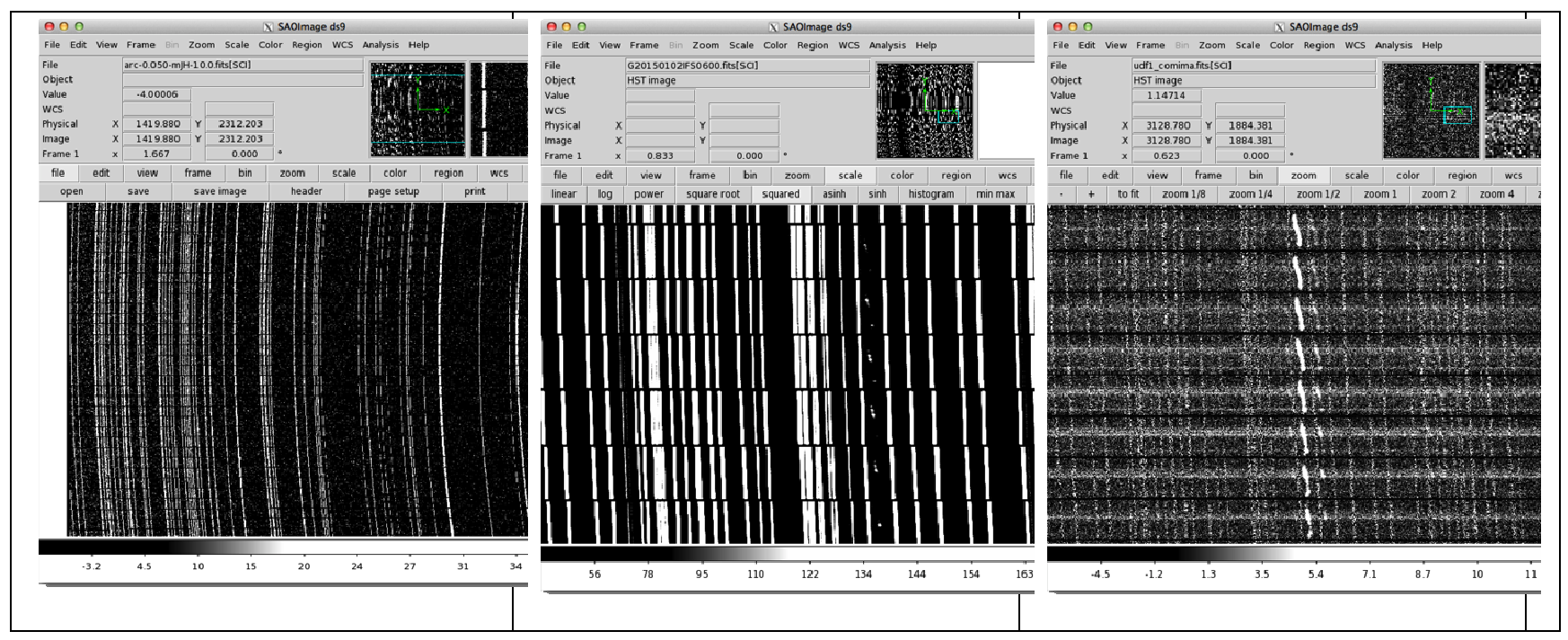

Figure 4: Simulated raw GMTIFS observations are used to assess instrument performance. A modified version of the PyWiFeS ${ }^{[13]}$ data processing system performs the necessary calibration steps to return scientifically useful data cubes. 


\subsection{Imager}

The GMTIFS Imager provides high-angular resolution imaging over a $20.4 \times 20.4$ arcsecond field well matched to the adaptive-optics corrected field of the single conjugate LTAO system. The 5 mas pixels sample the 10 mas diffraction limit at $\lambda \sim 1 \mu \mathrm{m}$. The marginal oversampling the longer wavelengths is of limited consequence as most broad-band observations will be largely background limited. A standard suite of broadband filters will be available (Figure 5). The current filter wheel design favors two 10-position filter wheels leaving eight positions free for the inclusion of a small suite of narrow-band filters (once necessary clear, compensated and blocked positions are included).

As well as it's primary scientific function, the GMTIFS Imager provides a number of additional critical capabilities for GMTIFS

- It enables rapid and accurate target acquisition.

- Simultaneous imaging in the complementary band during IFS observations. This will assist in image alignment for dithered mosaics on faint sources and provides some additional contemporary PSF information.

- Provide a means to assess the None-Common Path (NPC) error between the OIWFS and the science arms of GMTIFS.

- On-Detector Guide Windows (ODGW) provide access to the central science field that is inaccessible to the OIWFS due to the adopted spatial split architecture for GMTIFS.

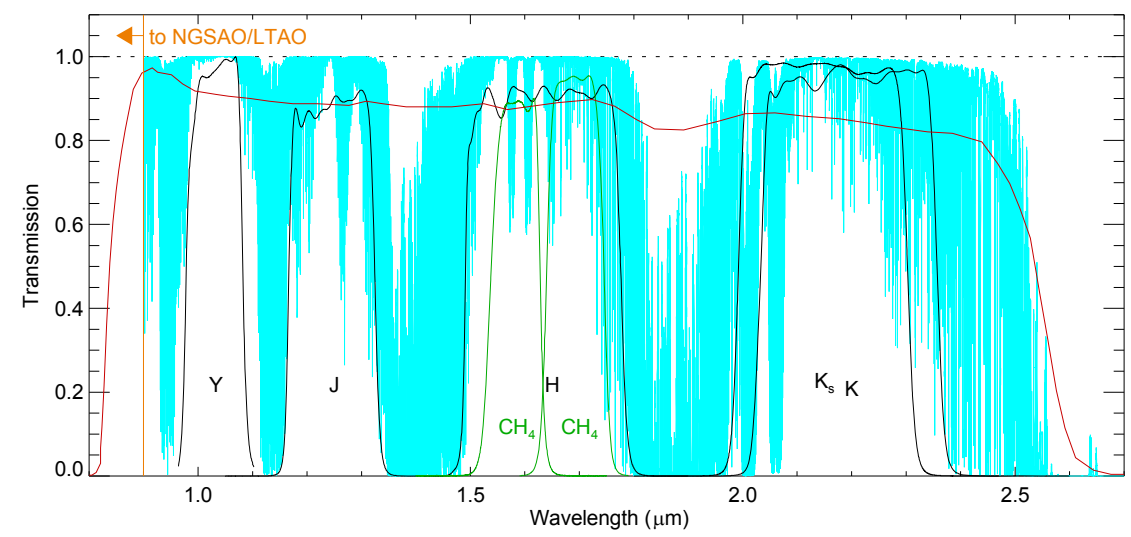

Figure 5 : The baseline GMTIFS Imager filter set is shown against the atmospheric transmission windows. Transmission data is taken from the Gemini-South template for Cerro Pachon: http://www.gemini.edu/sciops/telescopes-andsites/observing-condition-constraints/ir-transmission-spectra

\subsection{Adaptive optics operating modes}

The GMTIFS will operate with all modes of the GMT adaptive optics. While the high spatial sampling would make natural seeing operations very inefficient, recent analysis of the telescope phasing system suggests excellent ground layer correction will form part of the default phasing strategy for GMT and hence GLAO observations with GMTIFS will provide valuable early science opportunities and may well be the default calibration mode due to the higher assumed onsky efficiency.

Natural guide star AO will provide the very highest Strehl ratio observations on-axis for sources with suitable natural guide stars (with local differential flexure tracked by either the On-Instrument Wave-Front Sensor, OIWFS, or through the use of On-Detector Guide Windows, ODGW, within the science Imager). Simulations predict the very best performance will be available with a sky coverage of $>20 \%$. 
Laser Tomography AO will provide the workhorse extragalactic observation mode, for which modest Strehl and high ensquare energy are sufficient provided high sky coverage is achieved. Simulations indicate that a sky-coverage of $>$ $80 \%$ is straightforward, with Strehl $>0.3$, with a number of options for achieving higher sky-coverage currently under consideration. These include the use of the emerging electron Avalanche Photodiode (eAPD) ${ }^{[14,15,16]}$ detectors, and image sharpening of the off-axis guide star via a cooled $\mathrm{DM}^{[17]}$ in the OIWFS in an operating mode akin to MOAO operations.

\subsection{On-Instrument Wavefront Sensor and the Beam Steering Mirror feed}

The GMTIFS requires an On-Instrument Wavefront Sensor (OIWFS) to provide flexure and other low-order correction modes between the external active/adaptive-optics systems and the cold work surface on which the instrument is mounted with the cryostat. The OIWFS also moves focus and calibration information during LTAO observations. For historical reasons, the OIWFS was not part of the GMTIFS CoDR or the current PDS contract, with the preliminary design performed under contract to the GMT organization by a parallel team (also at the ANU). This rather unsatisfactory situation has recently been rectified with the OIWFS now adopted a GMTIFS subsystem (a primary recommendation of the CoDR review panel). The OIWFS feed receives the full wavelength range $(\lambda=0.95-2.5 \mu \mathrm{m})$ passed to the GMTIFS cryostat via a spatial split of the input field. The starlight is relayed from a star anywhere with the 180 arcsecond diameter guide field, excluding the central $20.4 \times 20.4$ arcsecond science field, via a fully steerable Beam Steering Mirror $\left(\mathrm{BSM}^{[8,9]}\right)$. As discussed above, the off-axis guide star image is sharpened by a cooled $\left(\mathrm{T} \approx-40^{\circ} \mathrm{C}\right)$ deformable mirrors ${ }^{[17]}$.

The preliminary design for the LTAO OIWFS required two Teledyne-H2RG detectors, operating in highly tuned readout modes. The evolving strategy for GMTO AO implementation in all modes, and the availability of extremely low readout noise eAPD detectors ${ }^{[16]}$ means that it is now appropriate for a significant review of the OIFWs concept. This work will be undertaken in 2016/2017 as part of the GMTIFS PDS.

Table 1: Overview of GMTIFS Parameters

\begin{tabular}{|c|c|c|}
\hline \multicolumn{3}{|c|}{ Integral Field Spectrograph } \\
\hline Spaxel size & $\begin{array}{c}\text { Angular resolution } \\
\text { element }\end{array}$ & Field of view \\
\hline 50 mas & $100 \mathrm{mas}$ & $4.40 \times 2.25$ \\
\hline 25 mas & 50 mas & $2.20 \times 1.125$ \\
\hline 12 mas & 24 mas & $1.056 \times 0.54$ \\
\hline 6 mas & 12 mas & $0.528 \times 0.27$ \\
\hline \multirow{2}{*}{$\begin{array}{l}\text { Resolving } \\
\text { power }\end{array}$} & $\mathrm{R} \sim 5,000$ & $\begin{array}{l}0.95-1.35 \mu \mathrm{m}(\mathrm{m} Y J) \\
1.19-1.80 \mu \mathrm{m}(\mathrm{m} J H) \\
1.64-2.49 \mu \mathrm{m}(\mathrm{m} H K)\end{array}$ \\
\hline & $\mathrm{R} \sim 10,000$ & $\begin{array}{l}0.95-1.135 \mu \mathrm{m}(\mathrm{h} Y) \\
1.10-1.35 \mu \mathrm{m}(\mathrm{h} J) \\
1.47-1.80 \mu \mathrm{m}(\mathrm{hH}) \\
2.04-2.51 \mu \mathrm{m}(\mathrm{h} K)\end{array}$ \\
\hline \multicolumn{3}{|c|}{ Imager } \\
\hline Field of view & $20.4 \times 20.4 \operatorname{arcsec}$ & \\
\hline Pixel size & 5 mas & \\
\hline Filter set & Two 10 position wheels & \\
\hline
\end{tabular}




\begin{tabular}{|c|c|}
\hline & $\begin{array}{l}\text { - } 2 \text { blanking plates, } 2 \text { clear positions, } 1 \text { path length compensation for broad-band ADC } \\
\text { calibration } \\
\text { - } \text { Broad band } Y J K_{s} K \\
\text { - } \quad \text { Medium band } \mathrm{CH}_{4} \text { pair } \\
\text { - } \quad \text { Narrow band to be confirmed }\end{array}$ \\
\hline Utility wheel & 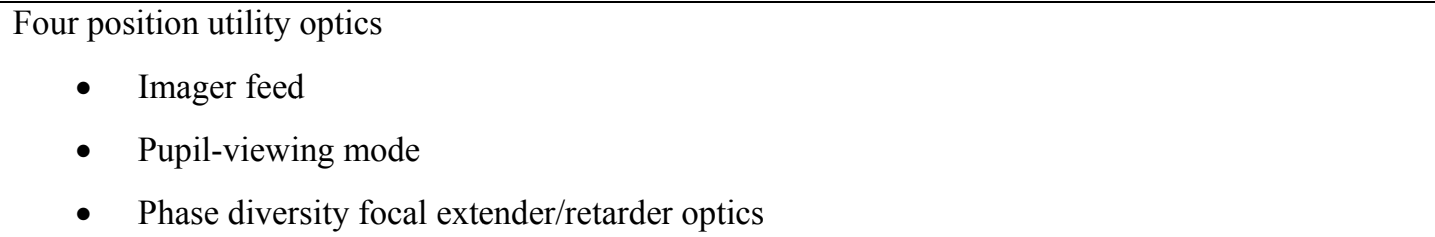 \\
\hline
\end{tabular}

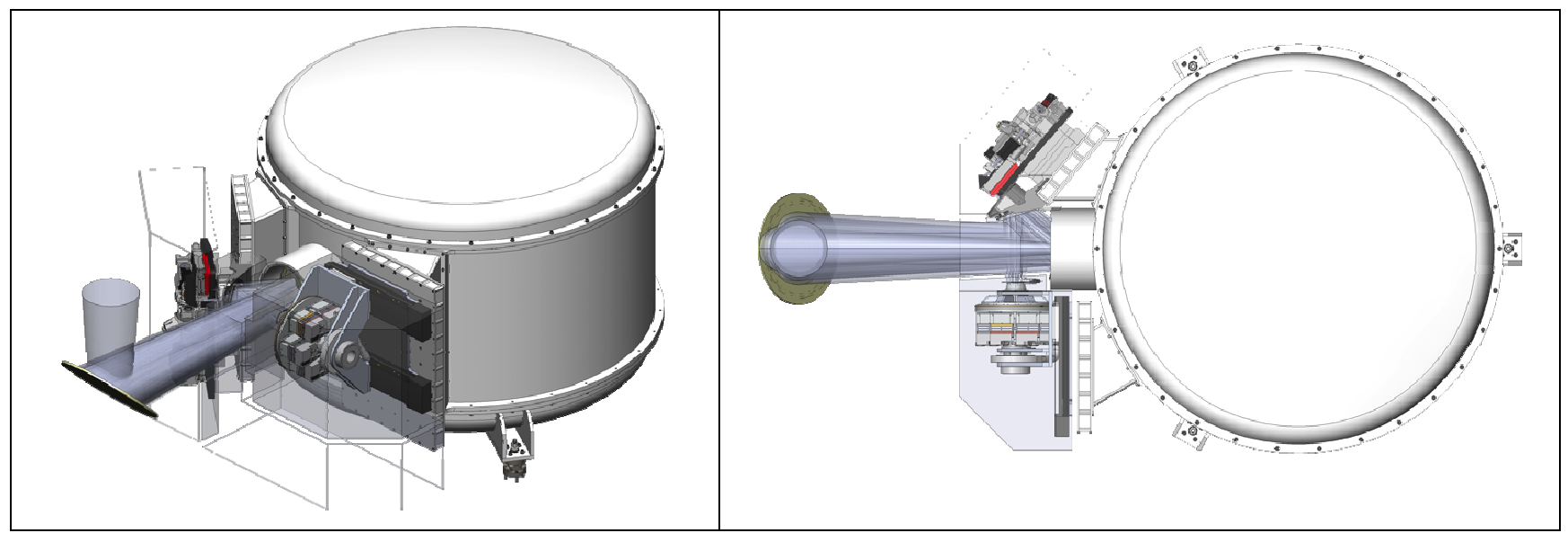

Figure 6: A front and plan view of the GMTIFS cryostat shows the GMT tertiary mirror folding the 180 arcsecond diameter field to GMTIFS. The dichroic cryostat window reflects wavelengths $\lambda<0.95 \mu \mathrm{m}$ to the external NGSAO and LTAO wavefront sensors mounted to the front of the cryostat.

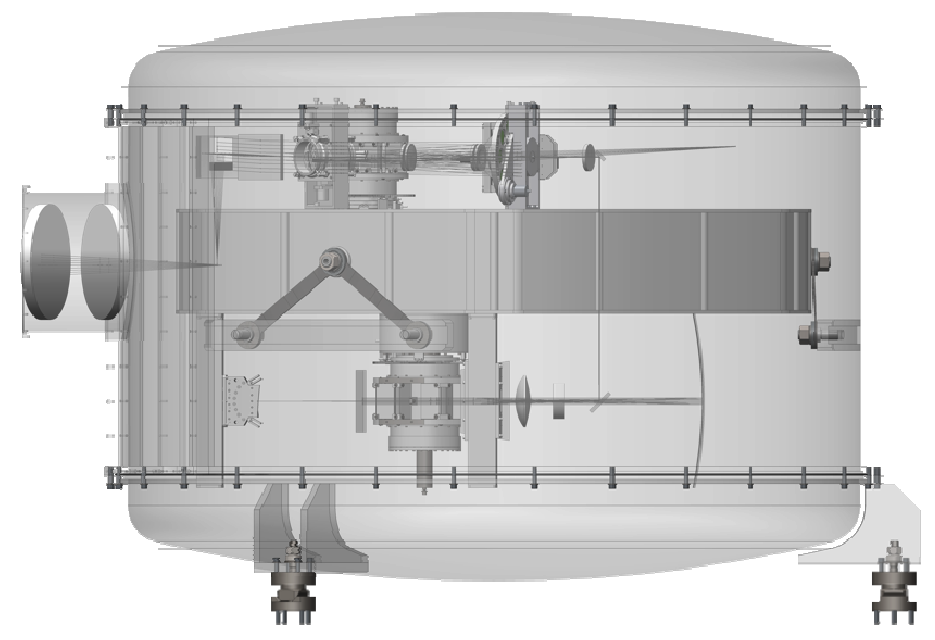

Figure 7: Partially populated optical table layout within the reduced cryostat volume. Final design for all optical elements is complete with some mechanical mounts awaiting tolerancing as part of the Preliminary Design Study. The central chamber of the cold work surface houses the OIWFS beam steering mirror, while the fore optics, Imager and IFS plus OIWFS are distributed across the upper and lower plains. 


\section{DESIGN EVOLUTION WITH THE PRELIMINARY DESIGN STUDY}

A number of elements of the instrument design presented at the Conceptual Design Review (CoDR) have evolved significantly as part of the Preliminary Design Study. These evolutions are focused on mitigating design risk while ensuring design requirements are met and reducing instrument budgets (mass, volume, cost) where possible

\section{IFS refractive camera}

A primary concern at the CoDR was the manufacturability of the Three Mirror Anastigmat (TMA) employed as the camera system for the IFS. While the TMA system delivered excellent instrument transmission, the mirror surfaces placed demanding tolerances on surface roughness for wavefront error control an the largest element was beyond the manufacturing capabilities of our preferred vendors at that time. Through an internal trade study, an all refractive IFS camera design was selected as the new baseline for GMTIFS (Figure 8).

The demanding specification for the camera leads to some novel glass selections. The use of, state of the art aspheres on the two crystal materials has enabled the implementation of only 4 elements for the camera design. The first element is $\mathrm{BaF}_{2}$ (Barium Fluoride, single crystal) with an asphere on the concave side. The second and third elements are Ohara S$\mathrm{NPH} 2$ glass and $\mathrm{CaF}_{2}$ (Calcium Fluoride, single crystal), with an asphere on the second convex face. The final element is IR grade fused silica (Infrasil 302).

The Ohara Glass S-NPH2, is a novel selection, in that it has not to our knowledge been used in cryogenic near-infrared instruments in the past. Although there is some internal absorption beyond $2 \mu \mathrm{m}$, the internal transmission out to $2.5 \mu \mathrm{m}$ is still acceptable. Indeed, the modest losses in transmission inherent in the switch from the TMA to the refractive camera design are largely offset by gains in other areas, leading to a PDS instrument transmission little changed from that proposed at the CoDR. Custom measurement of both the cryogenic refractive index and the cryogenic thermal strain will be sourced during the PDS to allow completion the final cold optical design at the Critical Design stage. ANU is working in partnership with the Nikon Glass Business Unit on tolerancing the current design.

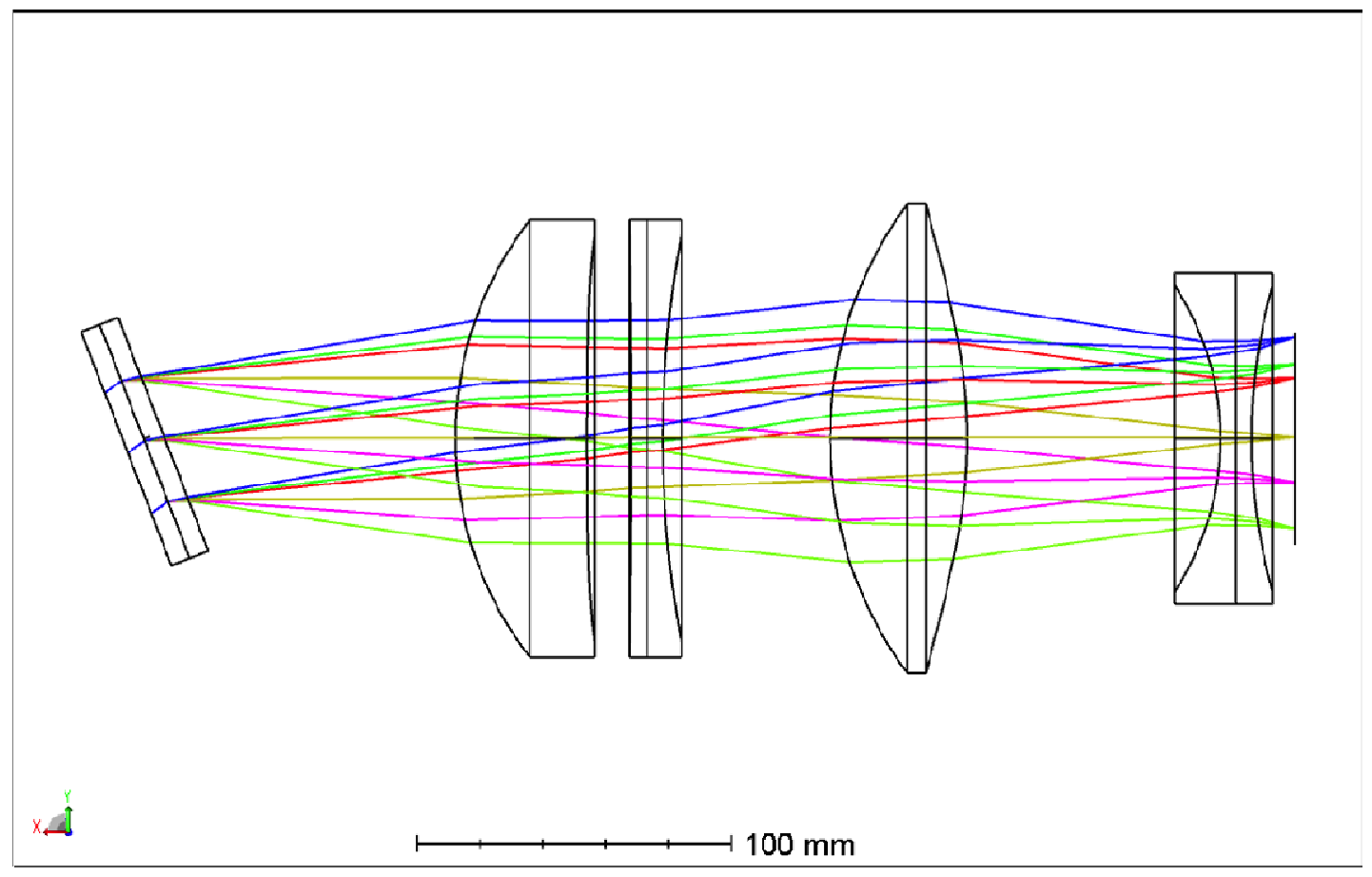

Figure 8: A fully refractive camera design for the IFS replaces the earlier reflective TMA due to concerns over the manufacturability of the TMA elements. 


\subsection{Atmospheric dispersion correction}

An atmospheric dispersion corrector is deemed necessary for two reasons. Firstly, such correction is critical to achieve broad-band imaging at the GMT diffraction limit since differential refraction across the conventional astronomical passbands would introduce significant image blur at even modest zenith distances. Secondly, while integral-field spectroscopy does in principle allow for correction for refraction in post observation image processing, the significant differential refraction effectively reduces the field-of-view with common wavelength coverage. Increasing the IFS field of view requires significant expense in terms of detector real-estate and hence proper dispersion correction is deemed essential.

An initial analysis for the CoDR indicated that no significant saving in cost or complexity could be gained from singleband only correction (i.e., correcting across only a single atmospheric pass-band at a time) while delivering the required image quality (single band correction would also precludes the simultaneous use of the Imager and IFS). Hence an ADC concept delivering full wavelength range correction $(\lambda=0.95-2.5 \mu \mathrm{m})$ was deemed necessary. Significant light losses were incurred from the 16 surface of the 8 element CoDR ADC concept. Additionally, it was also realized this concept introduced a highly variable transmission with field angle due to variation in the optical path-length for material absorption within the converging beam design. A significant redesign was therefor undertaken to move the ADC to a parallel beam, replacing the three element Offner relay and reflective cold stop with a two element off-axis parabola relay (OAP) with a transmissive cold stop. The transition to a parallel beam at the ADC allowed a simplified 6 element design. The fabrication, alignment and tolerance specification for this optic is currently under review in partnership with the with Glass Business Unit at Nikon.

The high refractive index glasses proposed for the six-element ADC (S-LAH71, S-FPL51, and, S-FPM2) produce a mild degradation in transmission at wavelengths $\lambda>2.2 \mu \mathrm{m}$ (particularly the use of S-LAH71), $\sim 4 \%$ relative to the $K$-band average transmission. Even with the inclusion of this loss, the overall instrument transmission is improved significantly over the CoDR design due to the lower number of surfaces in the ADC. Packaging of the two element OAP relay requires an additional fold mirror compared to the three element Offner relay and so there is no saving in reflective surfaces, however a significant saving in wavefront error is achieved since the large beam footprint on the second element of the Offner really had been the dominant source of wavefront error in the CoDR concept.

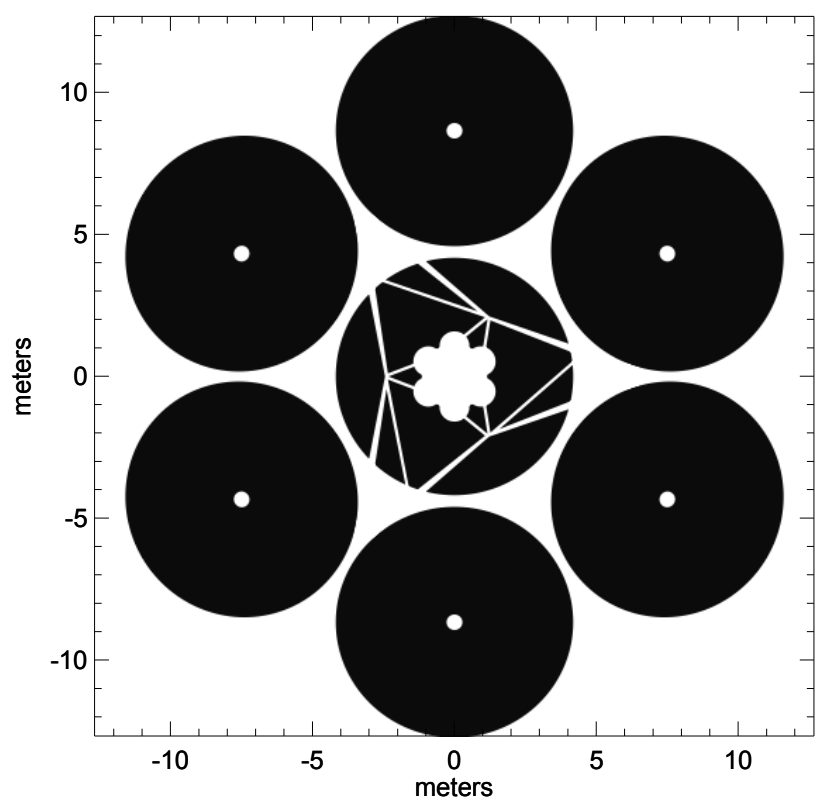

Figure 9: A representation of the GMT segmented pupil is shown. The transmissive GMTIFS cold stop map to the 7 M1 segments. The detailed design of the central obstruction is dependent on the final GMT ASM package design. The central support hubs for the outer ASM segments will not be masked. The GMTIFS pupil image quality is matched to the diffraction limit from the field stop provided by the $\sim 20.4 \times 20.4$ arcsec science pick off mirror. 


\subsection{Transmission}

The Conceptual Design Study specification for the GMTIFS specified an instrument throughput of $\geq 30 \%$. The Preliminary Design Study concept meets or exceeds this original specification at all wavelengths and in all modes of operations. The instrument throughput budget, excluding the detector quantum efficiency, telescope and atmospheric transmission, is given in Table 2 .

Table 2: Instrument transmission - Telescope and atmospheric transmission are excluded here, as is detector QE.

\begin{tabular}{|l|c|c|c|c|}
\hline \multicolumn{5}{|c|}{ Preliminary Design Study: Transmission Estimation } \\
\hline Wavelength range & $\boldsymbol{Y}$ & $\boldsymbol{J}$ & $\boldsymbol{H}$ & $\boldsymbol{K}$ \\
\hline IFS (R 10,000) & 0.326 & 0.353 & 0.386 & 0.352 \\
\hline IFS (R 5,000) & 0.369 & 0.429 & 0.465 & 0.425 \\
\hline Imager & 0.457 & 0.519 & 0.549 & 0.473 \\
\hline
\end{tabular}

Table 3: The relative noise contribution from each source are assessed as a function of wavelength range. Source shot-noise is not considered. Contributions are in electrons per exposure at the stated integration time.

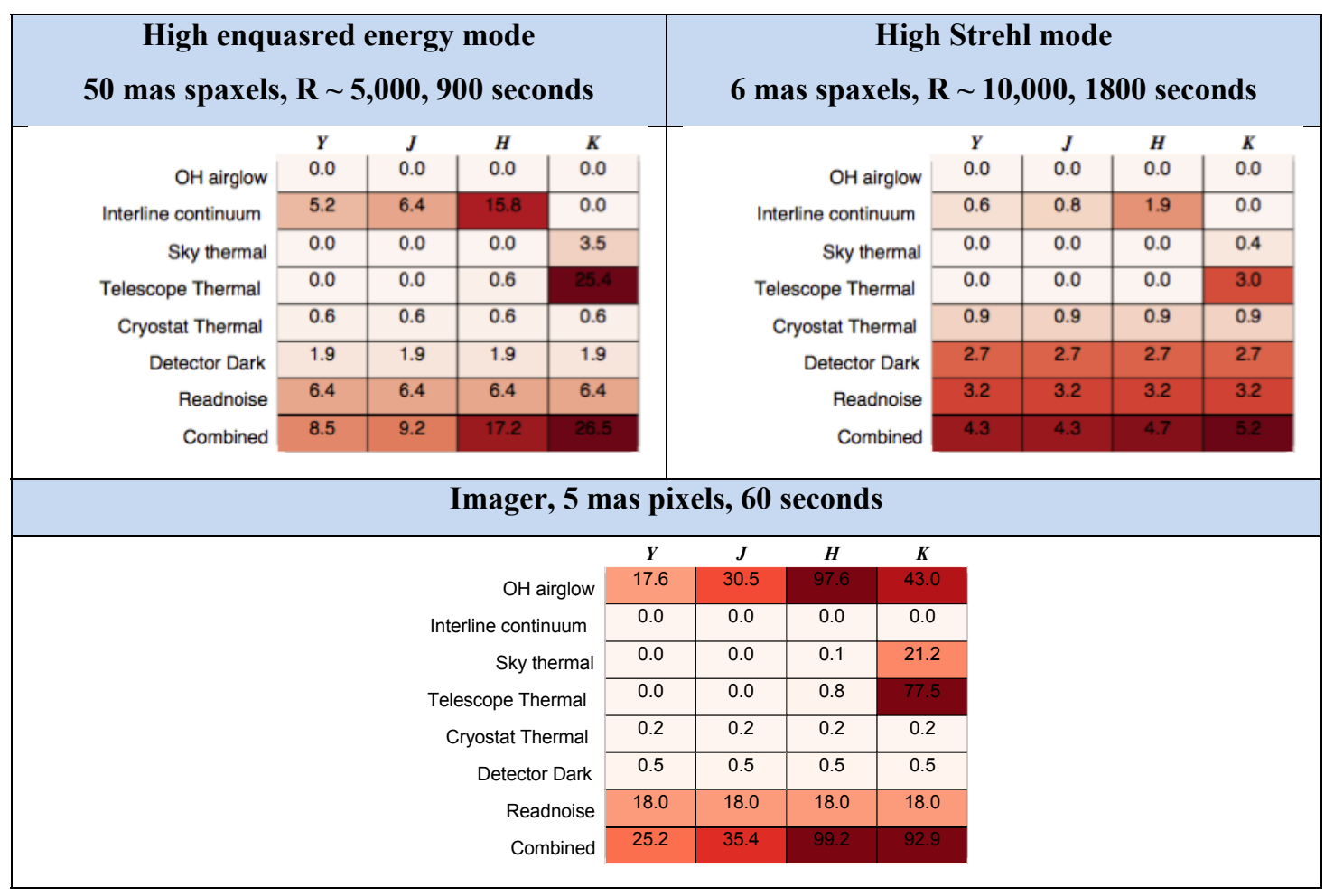

\subsection{Cryostat Volume Reduction}

With the removal of the TMA camera for the IFS, the largest element of which drove the height of the cryostat, in favor of a fully refractive camera and the adoption of an OAP relay rather than the Offner system, a significant reduction in cryostat volume has proven possible. Without the inclusion of any additional fold mirrors (beyond those replacing surfaces removed from the original design) the cryostat volume has been reduced to two thirds of the CoDR concept volume. This reduction not only results in a modest saving on physical hardware but more importantly provides a significant reduction in required cooling power to maintain a stable thermal environment. 
Furthermore, the size reduction also offers the opportunity for a regularization of the cryostat design. The largely triangular design of the earlier concept ${ }^{[1]}$ suffered significant distortion of the five large flat faces (three walls, top and bottom), requiring significant stiffening that add weight. The more regular layout of the updated design allows the adoption of a more conventional cylinder with hemispheric end caps. Such a design can achieve the requires stiffness with significantly less weight. The new packaging concept is shown in Figure 6, Figure 9 and Figure 10.

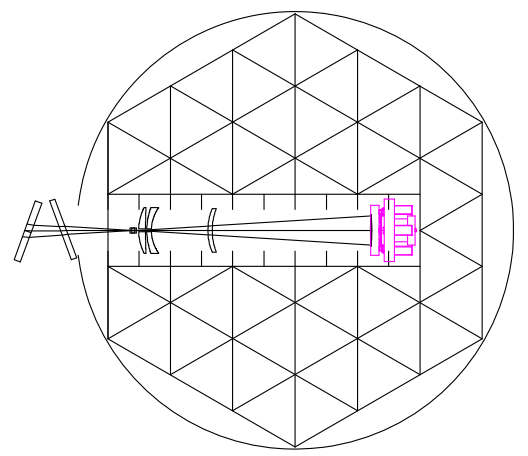

Mid Chamber

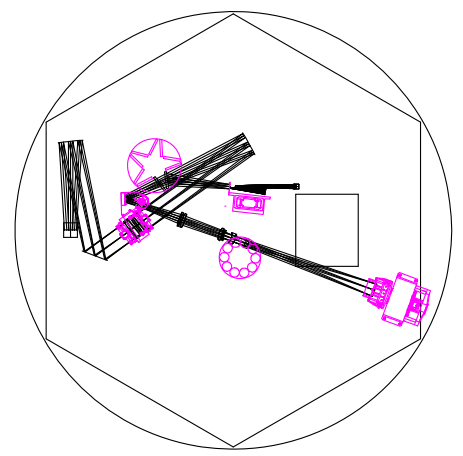

Upper Chamber

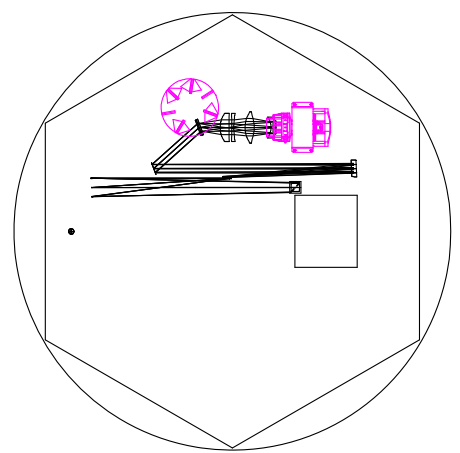

Lower Chamber

Figure 10: The folded optical system packaged into the reduced volume cryostat. The lower clear region in the upper chamber is reserved for the On-Instrument Wavefront Sensor (OIWFS).

\section{NEAR-INFRARED DETECTORS}

\subsection{Detector format}

A key remaining area of concern for GMTIFS is the availability of suitable large format high performance near-infrared detector arrays. The baseline design assumes two Teledyne $4 \mathrm{k} \times 4 \mathrm{k}$ H4RG-15 $\mu \mathrm{m}$ detectors, one at each of the science foci, the Imager and IFS instrument arms. While this detector complement is modest by the standards of many other ELT instruments, detector specifications are critical particularly so for the IFS as it will operate close to the readnoise limit in any modes (see

Table 3). Physically smaller arrays, e.g., the H4RG-10 $\mu$ m detectors proposed for extensive use with space telescope instrumentation would force the IFS to exceedingly fast optics that are likely unmanufacturable if the large spaxel scales and wide fields of view (necessary for low-surface brightness targets such as intermediate redshift galaxies) are to be maintained. Larger detector pixels, e.g., those of the Teledyne H2RG-18 $\mu$ m detectors deployed in the $2 \times 2$ mosaic found in the GSAOI ${ }^{[4]}$ camera, would be acceptable, but require a mosaic detector with significant array gaps $(\sim 5 \%$ of the linear size of the focal plane in the case of the GSAOI instrument). While such a mosaic gap can be tolerated in the spatial direction for the IFS split with appropriate fanning of the image slicer, no simple solution would prevent the loss of wavelength coverage for inter-array gaps in a mosaic focal plane. The fixed format of the proposed optical design, and the modest slit curvature, means significant wavelength regions (and hence redshift space for key extragalactic studies) would be lost to an instrument equipped with a mosaic detector.

The slower speed of the Imager camera means that $10 \mu \mathrm{m}$ pixels can be accommodated with modest optical revision and hence present little concern. Indeed, the expected improved read noise performance of the $10 \mu \mathrm{m}$ pixels may make a smaller device preferable, with the caveat that the Imager will typically be operating in a background limited mode with little impact from read noise. Additionally, the added cost and complexity of carrying multiple array formats must be taken into account.

\subsection{Detector characteristics}

Detector performance remains key for science operations with most IFS modes approaching the readnoise limit even after extensive Fowler sampling. Imager performance is largely driven by detector quantum efficiency and hence cosmetic quality raises a number of interesting trade options. While ELT instrumentation should demand excellence in 
detector quality, a brief study indicates the Imager is more sensitive to large cluster-defects rather than the global cosmetic quality (due to the nature of on-source dithering strategies), while the IFS has significant tolerance for highly localized defects if a higher global quality can be achieved (due to the nature of the flux redistribution across the detector with the images slicer). An aggregation of key performance trades across GMT near-infrared instrumentation is being explored as a collective assessment of an available suite of detectors may allow significant mutually beneficial performance improvements across a range of instruments (in the absence of perfect detector).

\subsection{Detector control}

The baseline GMTIFS concepts at CoDR assumed the Teledyne SIDECAR-ASIC control system. However, the questionable on-going support for this system, along with disappointing performance during laboratory testing with current generation detectors, led GMTIFS to conclude that an alternative option will be required. Recent instrumentation delivered by the ANU (e.g., Gemini/NIFS ${ }^{[3]}$; Gemini/GSAOI ${ }^{[4]}$; ANU/WiFeS ${ }^{[5]}$ ) have relied on the SDSU ARC-series III controller. However, this venerable system is aging and in its current form cannot delver the full-frame rates necessary for large format near-infrared detectors (needed for extensive Fowler sampling for high cadence wavefront sensing). To address the longstanding issues of pick-up noise in the signal lines between cryogenic detector and external control hardware for signal digitization, we have developed a cryogenic preamplification stage ${ }^{[16]}$ currently operating in tandem with an SDSU controller, while we explore a number of promising options for a new technology controller.

\subsection{On-Instrument Wavefront Sensor detectors}

The On-Instrument Wavefront Sensor (OIWFS) performance is key to achieving high adaptive optics sky coverage. The baseline OIWFS design was developed early in the project and presented in 2011. The system requirements have evolved significantly in the intervening years and as such the OIWFS will be rebaselined in Q3/4 2016. The original concept was limited to the consideration of Teledyne $\mathrm{H} 2 \mathrm{RG}$ detectors as a risk mitigation activity. However, the now routine availability of high performance electron Avalanche Photo-Diode (eAPD) detectors such as the SAPHIRA ${ }^{[14,15,16]}$ array from SELEX, that are essential free of readout noise, changes the paradigm for the OIWFS. The baseline will evaluate this emerging technology based a results from our technology demonstrator program that will deploy a SAPHIRA array as Lucky Imager ${ }^{[16]}$ at the ANU 2.3, telescope in early 2017. 


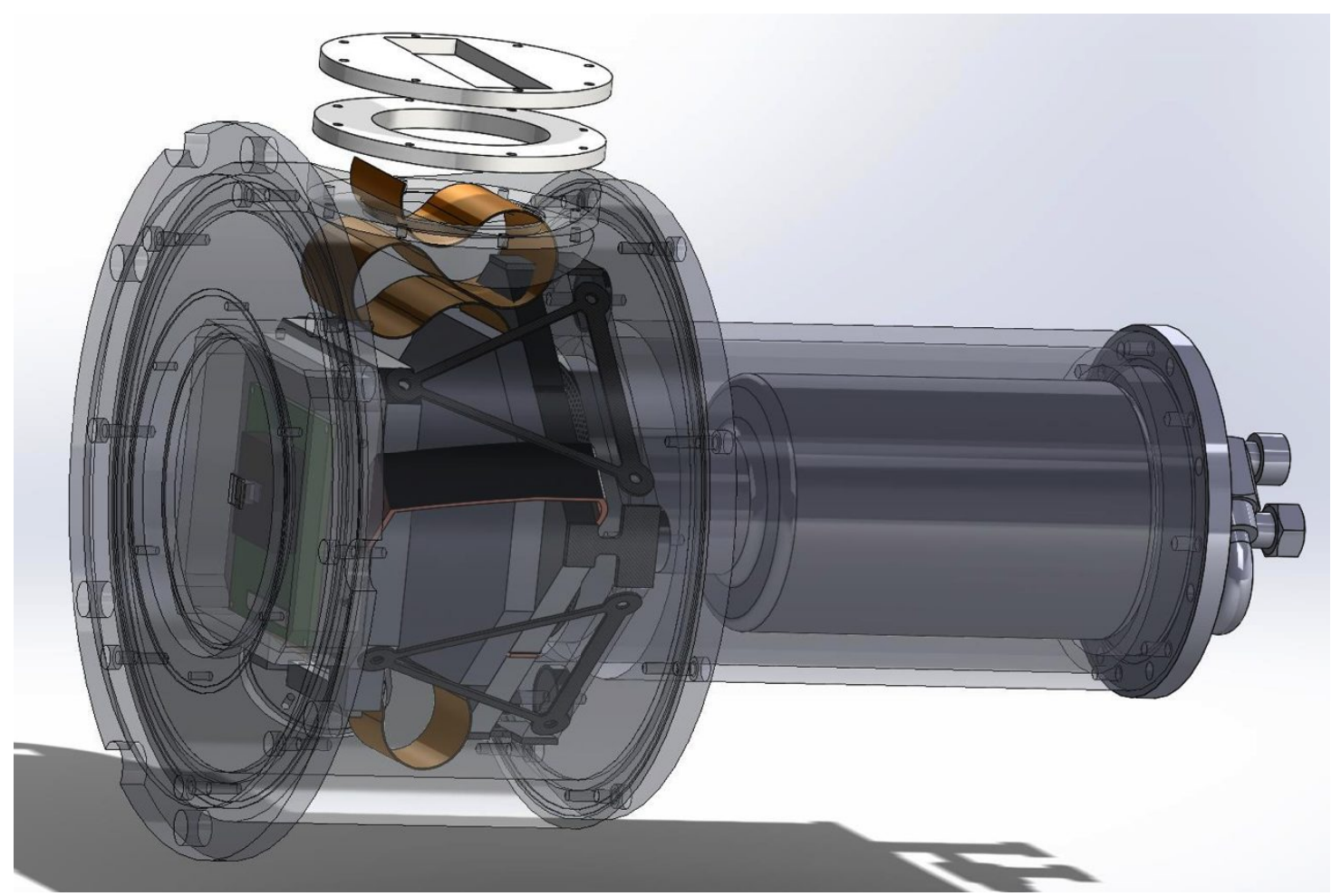

Figure 11: The SAPHIRA based Lucky Imager is to be delivered as a bare focal plane suitable for a range of high cadence imaging experiments. The basic cryostat concept builds upon a standardized design deployed at ANU with the WiFeS ${ }^{[5]}$ spectrograph.

\section{SUMMARY}

GMTIFS is a powerful new facility that will be the workhorse adaptive optics instrument in the early years of operation of the Giant Magellan Telescope. It builds on a long heritage of scientific instrumentation and will significantly extend studies of the physical process at work across a range of classes of astronomical source currently beyond the capabilities on 8-10 meter telescope systems. Wiley the design is technically challenging in some areas, risk mitigation through prototyping is addressing these concerns. The instrument concept stretches our capabilities and pushes new boundaries while retaining a firm foundation as a deliverable instrument for the new decade.

\section{ACKNOWLEDGEMENTS}

The work described in this manuscript would not have happened without the vision, drive and dedication of Professor Peter McGregor, whose unexpected death on March 2015 diminishes us all.

Australian membership in the GMT consortium is funded by the Department of Innovation, Industry, Science and Research, and Tertiary Education (DIISRTE) through the Australian Government Education Investment Fund (EIF). The GMTIFS Conceptual Design Study was funded by EIF, the Giant Magellan Telescope Organization (GMTO), and the National Science Foundation under Science Program No. 10 as issued for support of the Giant Segmented Mirror Telescope for the United States Astronomical Community in accordance with Proposal No. AST-0443999 submitted by AURA.

\section{REFERENCES}

1. McGregor,P. J., Bloxham, G. J., Boz, R., Davies, J., Doolan, M., Ellis, M., Hart, J., Jones, D. J., Luvaul, L., Nielsen, J., Parcell, S., Sharp, R. Stevanovic, D., Young, P. J., "GMT integral-field spectrograph (GMTIFS) conceptual design," Proc. SPIE, 8446, 1, (2012) 
2. Jacoby, G. H., Bouchez, A., Colless, M., DePoy,D., Fabricant, D., Hinz, P., Jaffe, D., Johns, M., McCarthy, P., McGregor, P., Shectman, S., Szentgyorgyi, A., "The instrument development and selection process for the Giant Magellan Telescope," Proc. SPIE, 8446, 1 (2012)

3. McGregor, P. J., Hart, J., Conroy, P. G., Pfitzner, M. L., Bloxham, G. J., Jones, D. J., Downing, M. D., Dawson, M., Young, P., Jarnyk, M., and Van Harmelen, J., "Gemini near-infrared integral field spectrograph (NIFS)," Proc. SPIE, 4841, 1581-1591, (2003)

4. McGregor, P.J., Hart, J., Stevanovic, D., Bloxham, G., Jones, D., van Harmelen, J., Griesbach, J., Dawson, M., Young, P., \& Jarnyk, M., "Gemini South Adaptive Optics Imager (GSAOI)," Proc. SPIE, 5492, 1033-1044 (2004)

5. Dopita, M., Hart, J., McGregor, P., Oates, P., Bloxham, G., Jones, D., "The Wide Field Spectrograph (WiFeS)," Ap\&SS, 310, 255-268 (2007).

6. Mukherjee, D., Bicknell, G., Sutherland, R., Wagner, A., "Relativistic jet feedback in high-redshift galaxies I: Dynamics," MNRAS, (in prep.)

7. Hart, J., Bloxham, G., Boz.,R., Bundy, D., Espeland, B., Fordham, B. , Hart, J., Nielsen, J., Sharp, R., Vaccarella, A., Vest, C., "GMTIFS: challenging optical design problems and their solutions for the GMT integral-field spectrograph," Proc. SPIE, this volume, (2016)

8. Sharp, R., Boz, R., Hart, J., Bloxham, G., Bundy, D., Davis, J., McGregor, P. J., Nielson, J., Vest, C., Young, P.J., "The adaptive optics beam steering mirror for the GMT Integral-Field Spectrograph, GMTIFS," Proc. SPIE, 9151, 0, (2014)

9. Davies, J., Bloxham, G., Boz, R., Bundy, D., Espeland, B., Fordham, B., Hart, J., Nielsen, J., Sharp, R., Vaccarella, A., Vest, C., Young, P.J., "GMTIFS: The Adaptive Optics Beam Steering Mirror for the GMT Integral-Field Spectrograph," Proc. SPIE, this volume (2016)

10. Hart, J., McGregor, P. J., Bloxham, G. J., "NIFS concentric integral field unit,” Proc. SPIE, 4841, 1581-1591 (2003)

11. Maihara, T., Iwamuro, F., Yamashita, T., Hall, D. N., Cowie, L. L., Tokunaga, A. T., Pickles, A. J., “Observations of the OH airglow emission," PASP, 691, 940-944. (1993)

12. Ellis, S. C., Bland-Hawthorn, J., "The case for OH suppression at near-infrared wavelengths," Monthly Notices of the Royal Astronomical Society, 386, 47-64 (2008)

13. Childress,M. J., Vogt, F. P. A., Nielsen, J., Sharp, R. G. "PyWiFeS: a rapid data reduction pipeline for the Wide Field Spectrograph (WiFeS)," Ap\&SS, 349, 617-636, (2014)

14. Finger, G., Baker, I., Alvarez, D., Ives, D., Mehrgan, L., Meyer, M., Stegmeier, J., Weller, H.J., "SAPHIRA detector for infrared wavefront sensing," Proc. SPIE, 9148, 16, (2014).

15. Atkinson, D., Hall, S., Baranec, C., Baker, I., Jacobson, S., Riddle, R., "Observatory deployment and characterization of SAPHIRA HgCdTe APD arrays," Proc. SPIE, 9154, 12, (2014)

16. Vaccarella, A., Sharp R., Ellis, M., Singh, S., Bloxham, G., Bouchez, A., Conan, R., Boz, R., Bundy, D., Davies, J., Espeland, B., Hart, J., Herrald, N., Ireland, M., Jacoby, G., Nielsen, J., Vest, C. , Young, P., Fordham, B., Zovaro, A., "Avalanche photo diodes in the observatory environment: lucky imaging at 1-2.5 microns," Proc. SPIE, this volume (2016)

17. Copeland, M., Price, I., Rigaut, F., Bloxham, G., Boz, R., Bundy, D., Espeland, B., Sharp, R., "GMTIFS: deformable mirror environmental testing for the on-instrument wavefront sensor," Proc. SPIE, this volume, (2016) 